\title{
Generalizing Choi-Like Maps
}

\author{
Dariusz Chruściński ${ }^{1}$ - Marcin Marciniak ${ }^{2}$ (D) . \\ Adam Rutkowski
}

Received: 1 February 2018 / Revised: 19 April 2018 / Accepted: 12 May 2018 /

Published online: 4 June 2018

(C) The Author(s) 2018

\begin{abstract}
A problem of further generalization of generalized Choi maps $\Phi_{[a, b, c]}$ acting on $\mathbb{M}_{3}$ introduced by Cho, Kye, and Lee is discussed. Some necessary conditions for positivity of the generalized maps are provided as well as some sufficient conditions. Also, some sufficient conditions for decomposability of these maps are shown.
\end{abstract}

Keywords Positive map · Indecomposable $\cdot$ Choi matrix $\cdot$ PPT $\cdot$ Separable state

Mathematics Subject Classification (2010) Primary 46L60 · 15B48 $\cdot$ 81P40 · Secondary 81Q10 - 46L05

\section{Introduction}

The paper concerns linear maps acting between matrix algebras. For $n \in \mathbb{N}$, by $\mathbb{M}_{n}$, we denote the algebra of square $n \times n$-matrices $X=\left(x_{i j}\right)$ with complex coefficients. Let $\Phi: \mathbb{M}_{m} \rightarrow \mathbb{M}_{n}$ be a linear map. We say that $\Phi$ is a positive map if $\Phi(X)$ is a positive definite matrix in $\mathbb{M}_{n}$ for every positive definite matrix $X \in \mathbb{M}_{m}$. Be reminded that for $k \in \mathbb{N}$, there are the following identifications: $\mathbb{M}_{k}\left(\mathbb{M}_{m}\right)=\mathbb{M}_{k} \otimes \mathbb{M}_{m}=\mathbb{M}_{k m}$, where

Marcin Marciniak

matmm@ug.edu.pl

Dariusz Chruściński

darch@fizyka.umk.pl

Adam Rutkowski

adam.rutkowski@ug.edu.pl

1 Institute of Physics, Nicolaus Copernicus University, Grudziądzka 5/7, 87-100 Toruń, Poland

2 Institute of Theoretical Physics and Astrophysics, Faculty of Mathematics, Physics and Informatics, University of Gdańsk, 80-309 Gdańsk, Poland 
$\mathbb{M}_{k}\left(\mathbb{M}_{m}\right)$ denotes the $C^{*}$-algebra of $k \times k$-matrices $\left(X_{i j}\right)$ with $X_{i j} \in \mathbb{M}_{m}$. Define a map $\Phi_{k}: \mathbb{M}_{k}\left(\mathbb{M}_{m}\right) \rightarrow \mathbb{M}_{k}\left(\mathbb{M}_{n}\right)$ by $\Phi_{k}\left(X_{i j}\right)=\left(\Phi\left(X_{i j}\right)\right)$. We say that the map $\Phi$ is $k$-positive if $\Phi_{k}$ is a positive map. If $\Phi$ is $k$-positive for every $k \in \mathbb{N}$, then it is called a completely positive map.

The transposition map $\theta_{n}: \mathbb{M}_{n} \rightarrow \mathbb{M}_{n}:\left(x_{i j}\right) \mapsto\left(x_{j i}\right)$ is known to be not completely positive map (even not 2-positive) [22]. We say that a map $\Phi: \mathbb{M}_{n} \rightarrow \mathbb{M}_{n}$ is $k$-copositive (respectively completely copositive) if the composition $\theta_{n} \circ \Phi$ is $k$-positive (respectively completely positive). A map $\Phi$ is said to be decomposable if it can be expressed as a sum $\Phi=\Phi_{1}+\Phi_{2}$, where $\Phi_{1}$ is a completely positive map while $\Phi_{2}$ is a completely copositive one. Otherwise, $\Phi$ is called indecomposable. For further details, we refer the reader to the book of Størmer [22].

The systematic study of positive maps on $\mathrm{C}^{*}$-algebras was started by a pioneering work of Erling Størmer in the 1960s of the last century [20]. Although the definitions are easy and the above notions seem to be rather elementary, the problem of description of all positive maps is still unsolved even in the case of low dimensional matrix algebras. In particular, still, there is no effective characterization of decomposable maps. In recent years, the importance of the theory of positive maps drastically increased because of its applications in mathematical physics, especially in rapidly emerging theory of quantum information [6, $10,13]$.

It was shown by Størmer [20] and Woronowicz [25] that every positive map $\Phi: \mathbb{M}_{2} \rightarrow$ $\mathbb{M}_{2}$ (Størmer) or $\Phi: \mathbb{M}_{2} \rightarrow \mathbb{M}_{3}$ (Woronowicz) is decomposable. The first example of an indecomposable positive map was given by Choi $[3,4]$. It is a map $\Phi: \mathbb{M}_{3} \rightarrow \mathbb{M}_{3}$ given by

$$
\Phi(X)=\left(\begin{array}{ccc}
x_{11}+x_{33} & -x_{12} & -x_{13} \\
-x_{21} & x_{22}+x_{11} & -x_{23} \\
-x_{31} & -x_{32} & x_{33}+x_{22}
\end{array}\right) \text {. }
$$

Although there is no systematic prescription of indecomposable maps, there are numerous examples scattered across literature [5, 7-9, 14-19, 23, 24].

An interesting approach to generalization of (1.1) was presented in [1]. For any triple of nonnegative numbers $a, b, c$ define a map $\Phi_{[a, b, c]}: \mathbb{M}_{3} \rightarrow \mathbb{M}_{3}$ by

$$
\Phi_{[a, b, c]}(X)=\left(\begin{array}{ccc}
a x_{11}+b x_{22}+c x_{33} & -x_{12} & -x_{13} \\
-x_{21} & c x_{11}+a x_{22}+b x_{33} & -x_{23} \\
-x_{31} & -x_{32} & b x_{11}+c x_{22}+a x_{33}
\end{array}\right),
$$

with $x_{i j}$ being the matrix elements of $X \in \mathbb{M}_{3}(\mathbb{C})$. One proves

Theorem 1.1 ([1]) The map $\Phi_{[a, b, c]}$ is positive but not completely positive if and only if

(1) $0 \leq a<2$,

(2) $a+b+c \geq 2$,

(3) if $a \leq 1$, then $b c \geq(1-a)^{2}$.

Moreover, being positive it is indecomposable if and only if $4 b c<(2-a)^{2}$.

Slightly different class was considered by Kye [12]

$$
\Phi_{\left[a ; c_{1}, c_{2}, c_{3}\right]}[X]=\left(\begin{array}{ccc}
a x_{11}+c_{1} x_{33} & -x_{12} & -x_{13} \\
-x_{21} & c_{2} x_{11}+a x_{22} & -x_{23} \\
-x_{31} & -x_{32} & c_{3} x_{22}+a x_{33}
\end{array}\right),
$$

with $a, c_{1}, c_{2}, c_{3} \geq 0$. He proved the following 
Theorem 1.2 A map $\Phi_{\left[a ; c_{1}, c_{2}, c_{3}\right]}$ is positive but not completely positive if and only if

(1) $1 \leq a<2$,

(2) $c_{1} c_{2} c_{3} \geq(2-a)^{3}$.

Moreover, $\Phi_{\left[a ; c_{1}, c_{2}, c_{3}\right]}$ is indecomposable and atomic.

Interestingly, Osaka shown

Theorem 1.3 ([17]) A map $\Phi_{\left[1 ; c_{1}, c_{2}, c_{3}\right]}$ is extremal if $c_{1} c_{2} c_{3}=1$.

Let us be reminded that for a linear map $\Phi: \mathbb{M}_{m} \rightarrow \mathbb{M}_{n}$, one can define its Choi matrix [2]. It is an element $C_{\Phi} \in \mathbb{M}_{m}\left(\mathbb{M}_{n}\right)$ defined by

$$
C_{\Phi}=\left(\Phi\left(E_{i j}\right)\right)
$$

where $\left\{E_{i j}: 1 \leq i, j \leq n\right\}$ is a system of matrix units in $\mathbb{M}_{m}$. The mapping $\Phi \mapsto C_{\Phi}$ is the so called Choi-Jamiołkowski isomorphism between $L\left(\mathbb{M}_{m}, \mathbb{M}_{n}\right)$ and $\mathbb{M}_{m}\left(\mathbb{M}_{n}\right)$ [11]. One has

Theorem 1.4 ([2]) A map $\Phi$ is completely positive if and only if its Choi matrix $C_{\Phi}$ is positive definite.

It turns out also that it is possible to characterize positivity and decomposability of $\Phi$ in terms of the matrix $C_{\Phi}$. To this end, let us recall that $C_{\Phi}$ can be considered as an element of the tensor product $\mathbb{M}_{m} \otimes \mathbb{M}_{n}$. An element $\rho \in M_{m} \otimes M_{n}$ is called a PPT matrix if both $\rho$ and $\left(\operatorname{id}_{\mathbb{M}_{m}} \otimes \theta_{n}\right)(\rho)$ are positive definite.

Theorem 1.5 ([10, 13, 21]) Let $\Phi$ be a linear map.

(i) $\Phi$ is positive if and only if

$$
\left\langle\xi \otimes \eta, C_{\Phi} \xi \otimes \eta\right\rangle \geq 0
$$

for every vectors $\xi \in \mathbb{C}^{m}, \eta \in \mathbb{C}^{n}$.

(ii) $\Phi$ is decomposable if and only if

$$
\operatorname{Tr}\left(\rho C_{\Phi}\right) \geq 0
$$

for every PPT matrix $\rho \in \mathbb{M}_{m} \otimes \mathbb{M}_{n}$.

The property (i) in the above theorem is called block-positivity [13].

\section{Choi-Like Maps}

In this paper, we consider the following generalization of the maps described in Introduction. Let

$$
A=\left(\begin{array}{lll}
a_{1} & b_{1} & c_{1} \\
c_{2} & a_{2} & b_{2} \\
b_{3} & c_{3} & a_{3}
\end{array}\right),
$$


with $a_{i}, b_{j}, c_{k} \geq 0$, and define $\Phi_{A}: \mathbb{M}_{3} \rightarrow \mathbb{M}_{3}$ as follows

$$
\Phi_{A}(X)=\left(\begin{array}{ccc}
a_{1} x_{11}+b_{1} x_{22}+c_{1} x_{33} & -x_{12} & -x_{13} \\
-x_{21} & c_{2} x_{11}+a_{2} x_{22}+b_{2} x_{33} & -x_{23} \\
-x_{31} & -x_{32} & b_{3} x_{11}+c_{3} x_{22}+a_{3} x_{33}
\end{array}\right) .
$$

Our aim is to describe some conditions for positivity of $\Phi_{A}$ as well as for decomposability.

Firstly, let us make the following observation.

Proposition 2.1 A map $\Phi_{A}$ is completely positive if and only if

$$
\left(\begin{array}{ccc}
a_{1} & -1 & -1 \\
-1 & a_{2} & -1 \\
-1 & -1 & a_{3}
\end{array}\right)
$$

is positive definite matrix.

Proof Observe that the Choi matrix of the map $\Phi_{A}$ is of the form

$$
C_{\Phi_{A}}=\left(\begin{array}{ccc|ccc|ccc}
a_{1} & \cdot & \cdot & \cdot & -1 & \cdot & \cdot & \cdot & -1 \\
\cdot & c_{2} & \cdot & \cdot & \cdot & \cdot & \cdot & \cdot & \cdot \\
\cdot & \cdot & b_{3} & \cdot & \cdot & \cdot & \cdot & \cdot & \cdot \\
\hline \cdot & \cdot & \cdot & b_{1} & \cdot & \cdot & \cdot & \cdot & \cdot \\
-1 & \cdot & \cdot & \cdot & a_{2} & \cdot & \cdot & \cdot & -1 \\
\cdot & \cdot & \cdot & \cdot & \cdot & c_{3} & \cdot & \cdot & \cdot \\
\hline \cdot & \cdot & \cdot & \cdot & \cdot & \cdot & c_{1} & \cdot & \cdot \\
\cdot & \cdot & \cdot & \cdot & \cdot & \cdot & \cdot & b_{2} & \cdot \\
-1 & \cdot & \cdot & \cdot & -1 & \cdot & \cdot & \cdot & a_{3}
\end{array}\right),
$$

where we used dots instead of zeros. Now, we apply Theorem 1.4. Since $b_{j}, c_{k}$ are nonnegative, positive definiteness of $C_{\Phi_{A}}$ is equivalent to positive definiteness of the submatrix (2.3).

Now, let us discuss some necessary conditions for positivity.

Theorem 2.2 A necessary condition for positivity of $\Phi_{A}$ is positivity of $\Phi_{[\bar{a}, \bar{b}, \bar{c}]}$, where

$$
\bar{a}=\frac{1}{3}\left(a_{1}+a_{2}+a_{3}\right), \quad \bar{b}=\frac{1}{3}\left(b_{1}+b_{2}+b_{3}\right), \quad \bar{c}=\frac{1}{3}\left(c_{1}+c_{2}+c_{3}\right) .
$$

Proof Let $\Phi_{A}$ be a positive map and consider a linear map

$$
\bar{\Phi}_{A}(X)=\frac{1}{3}\left(\Phi_{A}(X)+S \Phi_{A}\left(S^{*} X S\right) S^{*}+S^{*} \Phi_{A}\left(S X S^{*}\right) S\right),
$$

where $S$ denotes a unitary shift $S e_{i}=e_{i+1}$. It is clear that $\bar{\Phi}_{A}$ is positive. Moreover, $\bar{\Phi}_{A}=$ $\Phi[\bar{a}, \bar{b}, \bar{c}]$ with $\bar{a}, \bar{b}, \bar{c}$ defined in (2.4).

Let us introduce the following notation

$$
a_{*}=\left(a_{1} a_{2} a_{3}\right)^{1 / 3}, \quad b_{*}=\left(b_{1} b_{2} b_{3}\right)^{1 / 3}, \quad c_{*}=\left(c_{1} c_{2} c_{3}\right)^{1 / 3} .
$$

To find sufficient conditions, consider a positive map $\Phi[a, b, c]$ and define

$$
\Phi_{[a, b, c]}^{V}(X):=V^{-1 / 2} \Phi_{[a, b, c]}\left(V^{1 / 2} X V^{1 / 2}\right) V^{-1 / 2},
$$


where $V=\sum_{i=1}^{3} p_{i} E_{i i}$ with $p_{i}>0$. It is clear that $\Phi_{[a, b, c]}^{V}$ is positive. Moreover, one easily finds that $\Phi_{[a, b, c]}^{V}=\Phi_{A}$ with

$$
a_{i}=a_{*}, \quad b_{i}=\frac{p_{i+1}}{p_{i}} b, \quad c_{i}=\frac{p_{i+2}}{p_{i}} c, \quad i=1,2,3,
$$

that is, $A=V^{-1} A_{[a, b, c]} V$. Note that $b_{*}=b, c_{*}=c$, and $b_{i} c_{i+1}=b c$. Hence, one arrives at the following

Theorem 2.3 Assume that there exist $a, b, c$ and $V$ such that $\Phi_{[a, b, c]}$ is a positive map and all coefficients of the matrix

$$
M=A-V^{-1} A_{[a, b, c]} V,
$$

are non-negative numbers. Then $\Phi_{A}$ is a positive map.

Corollary 2.4 Assume that there exist $a, b, c \geq 0$ such that

(1) $\Phi_{[a, b, c]}$ defines a positive map,

(2) $\min \left\{a_{1}, a_{2}, a_{3}\right\} \geq a, b_{*} \geq b, c_{*} \geq c$,

(3) $b_{i} c_{i+1} \geq b c$ for $i=1,2,3$.

Then $\Phi_{A}$ is a positive map.

Proof We have $b_{1} b_{2} b_{3} \geq b^{3}$ and $c_{1} c_{2} c_{3} \geq c^{3}$. We construct a positive map with $b_{k}^{\prime}$ and $c_{k}^{\prime}$ such that $b_{k} \geq b_{k}^{\prime}$ and $c_{k} \geq c_{k}^{\prime}$. Let us take $p_{1}, p_{2}, p_{3}$ such that

$$
b_{1}=b_{1}^{\prime}:=\frac{p_{2}}{p_{1}} b, \quad b_{2}=b_{2}^{\prime}:=\frac{p_{3}}{p_{2}} b, \quad b_{3} \geq b_{3}^{\prime}:=\frac{p_{1}}{p_{3}} b .
$$

Clearly, $b_{1} b_{2} b_{3} \geq b_{1}^{\prime} b_{2}^{\prime} b_{3}^{\prime}=b^{3}$. Now, $b_{1} c_{2} \geq b c$ and hence, let us take $c_{2} \geq c_{2}^{\prime}:=\frac{p_{1}}{p_{2}} c$ and similarly for $c_{3}$ and $c_{1}$.

Now, the map with $b_{k}^{\prime}$ and $c_{k}^{\prime}$ is positive due to (2.5). Hence, the map with $b_{k}$ and $c_{k}$ is positive as well.

Remark 2.5 If $b_{1} b_{2} b_{3}=0$, then a sufficient condition reduces to

(1) $a_{1}, a_{2}, a_{3} \geq a$,

(2) $c_{1} c_{2} c_{3} \geq(2-a)^{3}$,

with $1 \leq a<2$. Interestingly, if $a_{1}=a_{2}=a_{3}=a$, then $a \geq 1$ and $c_{1} c_{2} c_{3} \geq(2-a)^{3}$ are necessary and sufficient [12]. Indeed, if $\Phi_{A}$ is positive and $c_{1} c_{2} c_{3}=c^{3}$ let us define $V$ by taking

$$
c_{1}=\frac{p_{3}}{p_{1}} c, \quad c_{2}=\frac{p_{1}}{p_{2}} c, \quad c_{3}=\frac{p_{2}}{p_{3}} c .
$$

Note the map $\Phi_{[a, b, c]}$ has to be positive and $b=0$, hence $a+c \geq 2$. Therefore, $a+$ $\sqrt[3]{c_{1} c_{2} c_{3}} \geq 2$ which implies $c_{1} c_{2} c_{3} \geq(2-a)^{3}$.

We end this section with the following remark.

Remark 2.6 Let us observe that condition (3) in Theorem 1.1 can equivalently be written as

$$
a+\sqrt{b c} \geq 1 \text {. }
$$


On the other hand, the condition (2) of Theorem 1.2 can be rewritten as

$$
a+c_{*} \geq 2
$$

Let us also remind that for a matrix

$$
A=\left(\begin{array}{ll}
a_{1} & b_{1} \\
b_{2} & a_{2}
\end{array}\right)
$$

one can define a map $\Phi_{A}: \mathbb{M}_{2} \rightarrow \mathbb{M}_{2}$ similarly to (2.2). It was shown by Kossakowski (see [6, Example 7.1]) that $\Phi_{A}$ is positive if and only if $a_{i} \geq 0, b_{i} \geq 0$ and

$$
\sqrt{a_{1} a_{2}}+\sqrt{b_{1} b_{2}} \geq 1
$$

Having all these observations in mind, one can conjecture that positivity of the map (2.2) is related to the following set of conditions:

$$
\begin{gathered}
\sqrt{a_{i} a_{i+1}}+\sqrt{b_{i} c_{i+1}} \geq 1, \\
a_{*}+b_{*}+c_{*} \geq 2 .
\end{gathered}
$$

In next sections, we shall discuss these conditions.

\section{Condition (2.6)}

Now, we consider further generalization. Let $A=\left(a_{i j}\right)_{i, j=1}^{n}$ be a matrix with nonnegative coefficients. Let us define a map $\Phi_{A}: \mathbb{M}_{n} \rightarrow \mathbb{M}_{n}$ by

$$
\Phi_{A}(X)=\Delta_{A}(X)-X,
$$

where

$$
\Delta_{A}(X)=\operatorname{diag}\left(\sum_{j=1}^{n} a_{1 j}^{\prime} x_{j j}, \sum_{j=1}^{n} a_{2 j}^{\prime} x_{j j}, \ldots, \sum_{j=1}^{n} a_{n j}^{\prime} x_{j j}\right)
$$

In the above formula $a_{i j}^{\prime}=a_{i j}+\delta_{i j}$, where $\delta_{i j}$ stands for the Kronecker delta.

Observe that positivity of $\Phi_{A}$ is equivalent to the inequality $\left\langle\eta, \Delta_{A}\left(\zeta \zeta^{*}\right) \eta\right\rangle \geq\left\langle\eta, \zeta \zeta^{*} \eta\right\rangle$ for every $\zeta, \eta \in \mathbb{C}^{n}$. One can easily show that the above is equivalent to

$$
\sum_{i, j=1}^{n} a_{i j}^{\prime} p_{i}^{2} q_{j}^{2} \geq\left(\sum_{i=1}^{n} p_{i} q_{i}\right)^{2}, \quad p_{i}, q_{i} \geq 0 .
$$

In the following main result of this section, we discuss the role of the condition (2.6). 
Theorem 3.1 Let $\Phi_{A}$ be given by (3.1). If the map $\Phi_{A}$ is positive, then

$$
\sqrt{a_{i i} a_{j j}}+\sqrt{a_{i j} a_{j i}} \geq 1, \quad i \neq j
$$

Proof Let $i \neq j$ be fixed. Observe that

$$
\begin{aligned}
& \sum_{k l} a_{k l}^{\prime} p_{k}^{2} q_{l}^{2}-\left(\sum_{k} p_{k} q_{k}\right)^{2} \\
= & \sum_{k l} a_{k l}^{\prime} p_{k}^{2} q_{l}^{2}-\sum_{k l} p_{k} p_{l} q_{k} q_{l} \\
= & \sum_{k} a_{k k} p_{k}^{2} q_{k}^{2}+\sum_{k<l}\left(a_{k l} p_{k}^{2} q_{l}^{2}+a_{l k} p_{l}^{2} q_{k}^{2}\right)-2 \sum_{k<l} p_{k} p_{l} q_{k} q_{l} \\
= & \sum_{k} a_{k k} p_{k}^{2} q_{k}^{2}+\sum_{k<l}\left(\sqrt{a_{k l}} p_{k} q_{l}-\sqrt{a_{l k}} p_{l} q_{k}\right)^{2}+2 \sum_{k<l}\left(\sqrt{a_{k l} a_{l k}}-1\right) p_{k} p_{l} q_{k} q_{l} .
\end{aligned}
$$

Consider the case $p_{k}=0$ and $q_{k}=0$ for every $k \notin\{i, j\}$. Then, the above expression is equal to

$$
\begin{aligned}
& a_{i i} p_{i}^{2} q_{i}^{2}+a_{j j} p_{j}^{2} q_{j}^{2}+\left(\sqrt{a_{i j}} p_{i} q_{j}-\sqrt{a_{j i}} p_{j} q_{i}\right)^{2}+2\left(\sqrt{a_{i j} a_{j i}}-1\right) p_{i} p_{j} q_{i} q_{j} \\
= & \left(\sqrt{a_{i i}} p_{i} q_{i}-\sqrt{a_{j j}} p_{j} q_{j}\right)^{2}+\left(\sqrt{a_{i j}} p_{i} q_{j}-\sqrt{a_{j i}} p_{j} q_{i}\right)^{2} \\
& +2\left(\sqrt{a_{i i} a_{j j}}+\sqrt{a_{i j} a_{j i}}-1\right) p_{i} p_{j} q_{i} q_{j} .
\end{aligned}
$$

Now, take

$$
p_{i}=q_{j}=1, \quad p_{j}=\left(\frac{a_{i j} a_{i i}}{a_{j i} a_{j j}}\right)^{1 / 4}, \quad q_{i}=\left(\frac{a_{i j} a_{j j}}{a_{j i} a_{i i}}\right)^{1 / 4} .
$$

Then, the expression from lines (3.7) and (3.8) reduces to

$$
2\left(\sqrt{a_{i i} a_{j j}}+\sqrt{a_{i j} a_{j i}}-1\right)\left(\frac{a_{i j}}{a_{j i}}\right)^{1 / 2} .
$$

It follows from the assumption that the expression (3.3) is nonnegative for every choice of $p_{k}, q_{k}$. Thus, we arrive at the inequality

$$
\sqrt{a_{i i} a_{j j}}+\sqrt{a_{i j} a_{j i}}-1 \geq 0
$$

which is equivalent to (3.2).

Having in mind, Theorem 1.1 one cannot expect that a converse theorem is also true. However, it turns out that a slight modification of (3.2) gives a sufficient condition for positivity and even decomposability.

\section{Theorem 3.2 If}

$$
\frac{1}{n-1} \sqrt{a_{i i} a_{j j}}+\sqrt{a_{i j} a_{j i}} \geq 1, \quad i \neq j
$$

then the map $\Phi_{A}$ is positive and decomposable. 
Proof Let us observe that

$$
\begin{aligned}
\sum_{i} a_{i i} p_{i}^{2} q_{i}^{2} & =\frac{1}{n-1} \sum_{i<j}\left(a_{i i} p_{i}^{2} q_{i}^{2}+a_{j j} p_{j}^{2} q_{j}^{2}\right) \\
& =\sum_{i<j}\left(\sqrt{\frac{a_{i i}}{n-1}} p_{i} q_{i}-\sqrt{\frac{a_{j j}}{n-1}} p_{j} q_{j}\right)^{2}+2 \sum_{i<j} \frac{\sqrt{a_{i i} a_{j j}}}{n-1} p_{i} p_{j} q_{i} q_{j} .
\end{aligned}
$$

According to the lines (3.3)-(3.6) and (3.10), one has

$$
\begin{aligned}
& \sum_{i j} a_{i j}^{\prime} p_{i}^{2} q_{j}^{2}-\left(\sum_{i} p_{i} q_{i}\right)^{2} \\
= & \sum_{i} a_{i i} p_{i}^{2} q_{i}^{2}+\sum_{i<j}\left(\sqrt{a_{i j}} p_{i} q_{j}-\sqrt{a_{j i}} p_{j} q_{i}\right)^{2}+2 \sum_{i<j}\left(\sqrt{a_{i j} a_{j i}}-1\right) p_{i} p_{j} q_{i} q_{j} \\
= & \sum_{i<j}\left(\sqrt{\frac{a_{i i}}{n-1}} p_{i} q_{i}-\sqrt{\frac{a_{j j}}{n-1}} p_{j} q_{j}\right)^{2}+\sum_{i<j}\left(\sqrt{a_{i j}} p_{i} q_{j}-\sqrt{a_{j i}} p_{j} q_{i}\right)^{2} \\
& +2 \sum_{i<j}\left(\frac{\sqrt{a_{i i} a_{j j}}}{n-1}+\sqrt{a_{i j} a_{j i}}-1\right) p_{i} p_{j} q_{i} q_{j} .
\end{aligned}
$$

It follows from (3.9) that the above expression is nonnegative for every nonnegative $p_{i}$ and $q_{i}$, so $\Phi_{A}$ is positive.

Now, we will show that $\Phi_{A}$ is decomposable. Let $\rho \in \mathbb{M}_{n}\left(\mathbb{M}_{n}\right)$ be a PPT matrix. We have $\rho=\left(\rho_{i j}\right)$, where $\rho_{i j} \in \mathbb{M}_{n}$. For $i \neq j$, let $r_{i j}$ be the $(i, j)$-coefficient of the matrix $\rho_{i j}$. For any $i, j$, let $\beta_{i j}$ denote the $j$-th diagonal term in the matrix $\rho_{i i}$. Thus, the matrix $\rho$ looks like

$$
\rho=\left(\begin{array}{ccccc|ccccc|c|ccccc}
\beta_{11} & * & * & \cdots & * & * & r_{12} & * & \cdots & * & \cdots & * & * & * & \cdots & r_{1 n} \\
* & \beta_{12} & * & \cdots & * & * & * & * & \cdots & * & \cdots & * & * & * & \cdots & * \\
* & * & \beta_{13} & \cdots & * & * & * & * & \cdots & * & \cdots & * & * & * & \cdots & * \\
\vdots & \vdots & \vdots & & \vdots & \vdots & \vdots & \vdots & & \vdots & & \vdots & \vdots & \vdots & & \vdots \\
* & * & * & \cdots & \beta_{1 n} & * & * & * & \cdots & * & \cdots & * & * & * & \cdots & * \\
\hline * & * & * & \cdots & * & \beta_{21} & * & * & \cdots & * & \cdots & * & * & * & \cdots & * \\
r_{21} & * & * & \cdots & * & * & \beta_{22} & * & \cdots & * & \cdots & * & * & * & \cdots & r_{2 n} \\
* & * & * & \cdots & * & * & * & \beta_{23} & \cdots & * & \cdots & * & * & * & \cdots & * \\
\vdots & \vdots & \vdots & & \vdots & \vdots & \vdots & \vdots & & \vdots & & \vdots & \vdots & \vdots & & \vdots \\
* & * & * & \cdots & * & * & * & * & \cdots & \beta_{2 n} & \cdots & * & * & * & \cdots & * \\
\hline \vdots & \vdots & \vdots & & \vdots & \vdots & \vdots & \vdots & & \vdots & & \vdots & \vdots & \vdots & & \vdots \\
\hline * & * & * & \cdots & * & * & * & * & \cdots & * & \cdots & \beta_{n 1} & * & * & \cdots & * \\
* & * & * & \cdots & * & * & * & * & \cdots & * & \cdots & * & \beta_{n 2} & * & \cdots & * \\
* & * & * & \cdots & * & * & * & * & \cdots & * & \cdots & * & * & \beta_{n 3} & \cdots & * \\
\vdots & \vdots & \vdots & & \vdots & \vdots & \vdots & \vdots & & \vdots & & \vdots & \vdots & \vdots & & \vdots \\
r_{n 1} & * & * & \cdots & * & * & r_{n 2} & * & \cdots & * & \cdots & * & * & * & \cdots & \beta_{n n}
\end{array}\right)
$$

while 


$$
\rho^{\Gamma}=\left(\begin{array}{ccccc|ccccc|c|ccccc}
\beta_{11} & * & * & \cdots & * & * & * & * & \cdots & * & \cdots & * & * & * & \cdots & * \\
* & \beta_{12} & * & \cdots & * & r_{12} & * & * & \cdots & * & \cdots & * & * & * & \cdots & * \\
* & * & \beta_{13} & \cdots & * & * & * & * & \cdots & * & \cdots & * & * & * & \cdots & * \\
\vdots & \vdots & \vdots & & \vdots & \vdots & \vdots & \vdots & & \vdots & & \vdots & \vdots & \vdots & & \vdots \\
* & * & * & \cdots & \beta_{1 n} & * & * & * & \cdots & * & \cdots & r_{1 n} & * & * & \cdots & * \\
\hline * & r_{21} & * & \cdots & * & \beta_{21} & * & * & \cdots & * & \cdots & * & * & * & \cdots & * \\
* & * & * & \cdots & * & * & \beta_{22} & * & \cdots & * & \cdots & * & * & * & \cdots & * \\
* & * & * & \cdots & * & * & * & \beta_{23} & \cdots & * & \cdots & * & * & * & \cdots & * \\
\vdots & \vdots & \vdots & & \vdots & \vdots & \vdots & \vdots & & \vdots & & \vdots & \vdots & \vdots & & \vdots \\
* & * & * & \cdots & * & * & * & * & \cdots & \beta_{2 n} & \cdots & * & r_{2 n} & * & \cdots & * \\
\hline \vdots & \vdots & \vdots & & \vdots & \vdots & \vdots & \vdots & & \vdots & & \vdots & \vdots & \vdots & & \vdots \\
\hline * & * & * & \cdots & r_{n 1} & * & * & * & \cdots & * & \cdots & \beta_{n 1} & * & * & \cdots & * \\
* & * & * & \cdots & * & * & * & * & \cdots & r_{n 2} & \cdots & * & \beta_{n 2} & * & \cdots & * \\
* & * & * & \cdots & * & * & * & * & \cdots & * & \cdots & * & * & \beta_{n 3} & \cdots & * \\
\vdots & \vdots & \vdots & & \vdots & \vdots & \vdots & \vdots & & \vdots & & \vdots & \vdots & \vdots & & \vdots \\
* & * & * & \cdots & * & * & * & * & \cdots & * & \cdots & * & * & * & \cdots & \beta_{n n}
\end{array}\right),
$$

where $\rho^{\Gamma}=\left(\theta_{n}\left(\rho_{i j}\right)\right)$ is the partially transposed $\rho$, and stars stand for any numbers. Since $\rho$ and $\rho^{\Gamma}$ are positive definite, $\beta_{i j} \geq 0, r_{j i}=\overline{r_{i j}}$, and

$$
\beta_{i i} \beta_{j j} \geq\left|r_{i j}\right|^{2}, \quad \beta_{i j} \beta_{j i} \geq\left|r_{i j}\right|^{2}, \quad i \neq j .
$$

Now, observe that

$$
\begin{aligned}
\operatorname{Tr}\left(\rho C_{\Phi_{A}}\right) & =\sum_{i, j=1}^{n} a_{i j} \beta_{i j}-2 \sum_{1 \leq i<j \leq n} \operatorname{Re}\left(r_{i j}\right) \\
& \geq \sum_{i=1}^{n} a_{i i} \beta_{i i}+\sum_{1 \leq i<j \leq n}\left(a_{i j} \beta_{i j}+a_{j i} \beta_{j i}\right)-2 \sum_{1 \leq i<j \leq n}\left|r_{i j}\right| \\
& =\frac{1}{n-1} \sum_{1 \leq i<j \leq n}\left(a_{i i} \beta_{i i}+a_{j j} \beta_{j j}\right)+\sum_{1 \leq i<j \leq n}\left(a_{i j} \beta_{i j}+a_{j i} \beta_{j i}\right)-2 \sum_{1 \leq i<j \leq n}\left|r_{i j}\right| \\
& \geq 2 \sum_{i<j}\left(\frac{1}{n-1} \sqrt{a_{i i} a_{j j} \beta_{i i} \beta_{j j}}+\sqrt{a_{i j} a_{j i} \beta_{i j} \beta_{j i}}-\left|r_{i j}\right|\right) \\
& \geq 2 \sum_{i<j}\left|r_{i j}\right|\left(\frac{1}{n-1} \sqrt{a_{i i} a_{j j}}+\sqrt{a_{i j} a_{j i}}-1\right) \geq 0 .
\end{aligned}
$$

The expression (3.12) was obtained by arithmetic-geometric mean inequality, while (3.13) is due to inequalities (3.11). Since $\rho$ is an arbitrary PPT matrix, we conclude that $\Phi_{A}$ is decomposable (cf. Theorem 1.5 (ii)).

For $n=2$, we immediately get the following corollary (cf. Remark 2.6).

Corollary 3.3 For $n=2$, a map $\phi_{A}$ is positive if and only if

$$
\sqrt{a_{11} a_{22}}+\sqrt{a_{12} a_{21}} \geq 1 \text {. }
$$


Remark 3.4 For $n=3$, the above theorem assures that the condition (2.6) is necessary for positivity of $\Phi_{A}$.

\section{Condition (2.7)}

Now, we are going to discuss necessity of condition (2.7). Let us start with the following

Theorem 4.1 Assume that the matrix (2.1) is of the form

$$
A=\left(\begin{array}{ccc}
a_{1} & b & c \\
c & a_{2} & b \\
b & c & a_{3}
\end{array}\right)
$$

for some arbitrary, $a_{1}, a_{2}, a_{3} \geq 1$ and $b, c>0$. If the map $\Phi_{A}$ given by (2.2) is positive, then

$$
\left(a_{1} a_{2} a_{3}\right)^{1 / 3}+b+c \geq 2 .
$$

Proof Observe that the Choi matrix of the map $\Phi_{A}$ is of the form

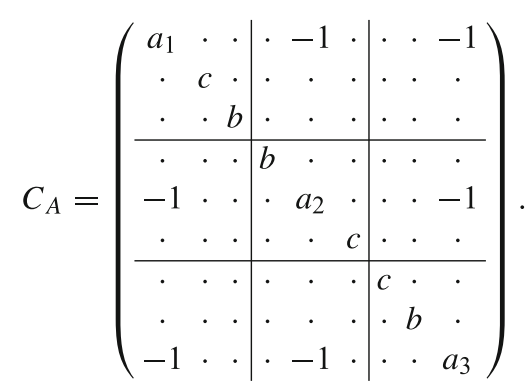

It follows from positivity of $\Phi_{A}$ that $C_{A}$ is block positive, i.e.,

$$
\left\langle\xi \otimes \eta, C_{A} \xi \otimes \eta\right\rangle \geq 0
$$

for every $\xi, \eta \in \mathbb{C}^{3}$.

Let

$$
\xi=\eta=\left(\left(a_{1}^{-1} a_{2} a_{3}\right)^{\frac{1}{12}},\left(a_{1} a_{2}^{-1} a_{3}\right)^{\frac{1}{12}},\left(a_{1} a_{2} a_{3}^{-1}\right)^{\frac{1}{12}}\right)^{\mathrm{T}} .
$$

Then,

$$
\xi \otimes \eta=\left(\left(a_{1}^{-1} a_{2} a_{3}\right)^{\frac{1}{6}}, a_{3}^{\frac{1}{6}}, a_{2}^{\frac{1}{6}}\left|a_{3}^{\frac{1}{6}},\left(a_{1} a_{2}^{-1} a_{3}\right)^{\frac{1}{6}}, a_{1}^{\frac{1}{6}}\right| a_{2}^{\frac{1}{6}}, a_{1}^{\frac{1}{6}},\left(a_{1} a_{2} a_{3}^{-1}\right)^{\frac{1}{6}}\right)^{\mathrm{T}} .
$$

Hence,

$\left\langle\xi \otimes \eta, C_{A} \xi \otimes \eta\right\rangle=a_{1}^{\frac{2}{3}} a_{2}^{\frac{1}{3}} a_{3}^{\frac{1}{3}}+a_{1}^{\frac{1}{3}} a_{2}^{\frac{2}{3}} a_{3}^{\frac{1}{3}}+a_{1}^{\frac{1}{3}} a_{2}^{\frac{1}{3}} a_{3}^{\frac{2}{3}}+b a_{3}^{\frac{1}{3}}+b a_{1}^{\frac{1}{3}}+b a_{2}^{\frac{1}{3}}$

$$
\begin{aligned}
& +c a_{2}^{\frac{1}{3}}+c a_{3}^{\frac{1}{3}}+c a_{1}^{\frac{1}{3}}-2 a_{3}^{\frac{1}{3}}-2 a_{1}^{\frac{1}{3}}-2 a_{2}^{\frac{1}{3}} \\
= & \left(a_{1}^{\frac{1}{3}}+a_{2}^{\frac{1}{3}}+a_{3}^{\frac{1}{3}}\right)\left(\left(a_{1} a_{2} a_{3}\right)^{\frac{1}{3}}+b+c-2\right) .
\end{aligned}
$$


Thus, inequality (4.3) leads to (4.2).

Remark 4.2 The vector $\xi$ in the above proof can be taken even simpler:

$$
\xi=\eta=\left(a_{1}^{-\frac{1}{6}}, a_{2}^{-\frac{1}{6}}, a_{3}^{-\frac{1}{6}}\right)^{\mathrm{T}} .
$$

Remark 4.3 For the case $n=2$, one can prove a similar result for

$$
A=\left(\begin{array}{ll}
a_{1} & b_{1} \\
b_{2} & a_{2}
\end{array}\right),
$$

where $a_{i} \geq 1, b_{i}>0$ are arbitrary. In this case, one takes

$$
\xi=\left(a_{1}^{-\frac{1}{4}} b_{2}^{\frac{1}{4}}, a_{2}^{-\frac{1}{4}} b_{1}^{\frac{1}{4}}\right)^{\mathrm{T}}, \quad \eta=\left(a_{1}^{-\frac{1}{4}} b_{2}^{-\frac{1}{4}}, a_{2}^{-\frac{1}{4}} b_{1}^{-\frac{1}{4}}\right)^{\mathrm{T}} .
$$

Theorem 4.4 Assume that the matrix (2.1) is of the form

$$
A=\left(\begin{array}{ccc}
a_{1} & b_{1} & 0 \\
0 & a_{2} & b_{2} \\
b_{3} & 0 & a_{3}
\end{array}\right)
$$

for some arbitrary, $a_{1}, a_{2}, a_{3} \geq 1$ and $b_{1}, b_{2}, b_{3}>0$. If the map $\Phi_{A}$ given by (2.2) is positive, then

$$
\left(a_{1} a_{2} a_{3}\right)^{1 / 3}+\left(b_{1} b_{2} b_{3}\right)^{1 / 3} \geq 2 .
$$

Proof The same idea as above. Observe that the Choi matrix of $\Phi_{A}$ has the form

$$
C_{A}=\left(\begin{array}{ccc|ccc|ccc}
a_{1} & \cdot & \cdot & \cdot & -1 & \cdot & \cdot & \cdot & -1 \\
\cdot & \cdot & \cdot & \cdot & \cdot & \cdot & \cdot & \cdot & \cdot \\
\cdot & \cdot & b_{3} & \cdot & \cdot & \cdot & \cdot & \cdot & \cdot \\
\hline \cdot & \cdot & \cdot & b_{1} & \cdot & \cdot & \cdot & \cdot & \cdot \\
-1 & \cdot & \cdot & \cdot & a_{2} & \cdot & \cdot & \cdot & -1 \\
\cdot & \cdot & \cdot & \cdot & \cdot & \cdot & \cdot & \cdot & \cdot \\
\hline \cdot & \cdot & \cdot & \cdot & \cdot & \cdot & \cdot & \cdot & \cdot \\
\cdot & \cdot & \cdot & \cdot & \cdot & \cdot & \cdot & b_{2} & \cdot \\
-1 & \cdot & \cdot & \cdot & -1 & \cdot & \cdot & \cdot & a_{3}
\end{array}\right) .
$$

Consider the following vectors $\xi$ and $\eta$ (see (4.4)):

$$
\xi=\left(\begin{array}{c}
a_{1}^{-\frac{1}{6}} b_{1}^{\frac{1}{6}} b_{3}^{-\frac{1}{6}} \\
a_{2}^{-\frac{1}{6}} b_{2}^{\frac{1}{6}} b_{1}^{-\frac{1}{6}} \\
a_{3}^{-\frac{1}{6}} b_{3}^{\frac{1}{6}} b_{2}^{-\frac{1}{6}}
\end{array}\right), \quad \eta=\left(\begin{array}{c}
a_{1}^{-\frac{1}{6}} b_{1}^{-\frac{1}{6}} b_{3}^{\frac{1}{6}} \\
a_{2}^{-\frac{1}{6}} b_{2}^{-\frac{1}{6}} b_{1}^{\frac{1}{6}} \\
a_{3}^{-\frac{1}{6}} b_{3}^{-\frac{1}{6}} b_{2}^{\frac{1}{6}}
\end{array}\right) .
$$


Then, the vector $\xi \otimes \eta$ is of the form

$$
\xi \otimes \eta=\left(\begin{array}{c}
a_{1}^{-\frac{1}{3}} \\
a_{1}^{-\frac{1}{6}} a_{2}^{-\frac{1}{6}} b_{1}^{\frac{1}{3}} b_{2}^{-\frac{1}{6}} b_{3}^{-\frac{1}{6}} \\
a_{1}^{-\frac{1}{6}} a_{3}^{-\frac{1}{6}} b_{1}^{\frac{1}{6}} b_{2}^{\frac{1}{6}} b_{3}^{-\frac{1}{3}} \\
a_{1}^{-\frac{1}{6}} a_{2}^{-\frac{1}{6}} b_{1}^{-\frac{1}{3}} b_{2}^{\frac{1}{6}} b_{3}^{\frac{1}{6}} \\
a_{2}^{-\frac{1}{3}} \\
a_{2}^{-\frac{1}{6}} a_{3}^{-\frac{1}{6}} b_{1}^{-\frac{1}{6}} b_{2}^{\frac{1}{3}} b_{3}^{-\frac{1}{6}} \\
a_{1}^{-\frac{1}{6}} a_{3}^{-\frac{1}{6}} b_{1}^{-\frac{1}{6}} b_{2}^{-\frac{1}{6}} b_{3}^{\frac{1}{3}} \\
a_{2}^{-\frac{1}{6}} a_{3}^{-\frac{1}{6}} b_{1}^{\frac{1}{6}} b_{2}^{-\frac{1}{3}} b_{3}^{\frac{1}{6}} \\
a_{3}^{-\frac{1}{3}}
\end{array}\right) .
$$

Like in the proof of Theorem 4.1, we calculate

$\left\langle\xi \otimes \eta, C_{A} \xi \otimes \eta\right\rangle$

$$
\begin{aligned}
= & a_{1}^{\frac{1}{3}}+a_{2}^{\frac{1}{3}}+a_{3}^{\frac{1}{3}}+a_{1}^{-\frac{1}{3}} a_{3}^{-\frac{1}{3}} b_{1}^{\frac{1}{3}} b_{2}^{\frac{1}{3}} b_{3}^{\frac{1}{3}}+a_{1}^{-\frac{1}{3}} a_{2}^{-\frac{1}{3}} b_{1}^{\frac{1}{3}} b_{2}^{\frac{1}{3}} b_{3}^{\frac{1}{3}}+a_{2}^{-\frac{1}{3}} a_{3}^{-\frac{1}{3}} b_{1}^{\frac{1}{3}} b_{2}^{\frac{1}{3}} b_{3}^{\frac{1}{3}} \\
& -2 a_{1}^{-\frac{1}{3}} a_{2}^{-\frac{1}{3}}-2 a_{1}^{-\frac{1}{3}} a_{3}^{-\frac{1}{3}}-2 a_{2}^{-\frac{1}{3}} a_{3}^{-\frac{1}{3}} \\
= & a_{1}^{\frac{1}{3}}+a_{2}^{\frac{1}{3}}+a_{3}^{\frac{1}{3}}+a_{2}^{\frac{1}{3}} a_{*}^{-1} b_{*}+a_{3}^{\frac{1}{3}} a_{*}^{-1} b_{*}+a_{1}^{\frac{1}{3}} a_{*}^{-1} b_{*}-2 a_{3}^{\frac{1}{3}} a_{*}^{-1}-2 a_{2}^{\frac{1}{3}} a_{*}^{-1}-2 a_{1}^{\frac{1}{3}} a_{*}^{-1} \\
= & a_{*}^{-1}\left(a_{1}^{\frac{1}{3}}+a_{2}^{\frac{1}{3}}+a_{3}^{\frac{1}{3}}\right)\left(a_{*}+b_{*}-2\right) .
\end{aligned}
$$

\section{Problem of Sufficiency}

Let us consider the matrix $A$ given by (4.1). We have shown that the following conditions (cf. (3.2), (4.2))

$$
\begin{gathered}
\left(a_{i} a_{i+1}\right)^{1 / 2}+(b c)^{1 / 2} \geq 1, \quad i=1,2,3, \\
\left(a_{1} a_{2} a_{3}\right)^{1 / 3}+b+c \geq 2
\end{gathered}
$$

are necessary for positivity of $\Phi_{A}$. For $a_{1}=a_{2}=a_{3}$, these condition are also sufficient [1]. Note that they are special cases of conditions (2.6) and (2.7).

However, these conditions are not sufficient for general case. Namely, we have the following no-go result.

Proposition 5.1 Let $a_{1}, a_{2}, a_{3}>0$ and $b \geq 0$ be such that

$$
\left(a_{1} a_{2} a_{3}\right)^{1 / 3}+b=2 .
$$


Let $A$ be the matrix in given by (4.1) with $c=0$. Then, $\Phi_{A}$ is positive if and only if $a_{1}=a_{2}=$ $a_{3}$.

Proof Sufficiency part follows from Theorem 1.1. We will show necessity. Assume that $\Phi_{A}$ is a positive map. Let

$$
\xi=\left(a_{1}^{-\frac{1}{6}} a_{3}^{\frac{1}{6}}, a_{2}^{-\frac{1}{6}} a_{1}^{\frac{1}{6}}, a_{3}^{-\frac{1}{6}} a_{2}^{\frac{1}{6}}\right)^{\mathrm{T}}
$$

Then,

$$
\xi \xi^{*}=\left(\begin{array}{ccccc}
a_{1}^{-\frac{1}{3}} a_{3}^{\frac{1}{3}} & a_{2}^{-\frac{1}{6}} a_{3}^{\frac{1}{6}} & a_{1}^{-\frac{1}{6}} a_{2}^{\frac{1}{6}} \\
a_{2}^{-\frac{1}{6}} a_{3}^{\frac{1}{6}} & a_{2}^{-\frac{1}{3}} a_{1}^{\frac{1}{3}} & a_{3}^{-\frac{1}{6}} a_{1}^{\frac{1}{6}} \\
a_{1}^{-\frac{1}{6}} a_{2}^{\frac{1}{6}} & a_{3}^{-\frac{1}{6}} a_{1}^{\frac{1}{6}} & a_{3}^{-\frac{1}{3}} a_{2}^{\frac{1}{3}}
\end{array}\right)
$$

and

$$
\Phi_{A}\left(\xi \xi^{*}\right)=\left(\begin{array}{ccc}
a_{1}^{\frac{2}{3}} a_{3}^{\frac{1}{3}}+b a_{2}^{-\frac{1}{3}} a_{1}^{\frac{1}{3}} & -a_{2}^{-\frac{1}{6}} a_{3}^{\frac{1}{6}} & -a_{1}^{-\frac{1}{6}} a_{2}^{\frac{1}{6}} \\
-a_{2}^{-\frac{1}{6}} a_{3}^{\frac{1}{6}} & a_{2}^{\frac{2}{3}} a_{1}^{\frac{1}{3}}+b a_{3}^{-\frac{1}{3}} a_{2}^{\frac{1}{3}} & -a_{3}^{-\frac{1}{6}} a_{1}^{\frac{1}{6}} \\
-a_{1}^{-\frac{1}{6}} a_{2}^{\frac{1}{6}} & -a_{3}^{-\frac{1}{6}} a_{1}^{\frac{1}{6}} & a_{3}^{\frac{2}{3}} a_{2}^{\frac{1}{3}}+b a_{1}^{-\frac{1}{3}} a_{3}^{\frac{1}{3}}
\end{array}\right) .
$$

Calculate the determinant of the above matrix

$$
\begin{aligned}
& \operatorname{det} \Phi_{A}\left(\xi \xi^{*}\right) \\
= & \left(a_{1}^{\frac{2}{3}} a_{3}^{\frac{1}{3}}+b a_{2}^{-\frac{1}{3}} a_{1}^{\frac{1}{3}}\right)\left(a_{2}^{\frac{2}{3}} a_{1}^{\frac{1}{3}}+b a_{3}^{-\frac{1}{3}} a_{2}^{\frac{1}{3}}\right)\left(a_{3}^{\frac{2}{3}} a_{2}^{\frac{1}{3}}+b a_{1}^{-\frac{1}{3}} a_{3}^{\frac{1}{3}}\right)-2 \\
& -a_{1}^{-\frac{1}{3}} a_{2}^{\frac{1}{3}}\left(a_{2}^{\frac{2}{3}} a_{1}^{\frac{1}{3}}+b a_{3}^{-\frac{1}{3}} a_{2}^{\frac{1}{3}}\right)-a_{3}^{-\frac{1}{3}} a_{1}^{\frac{1}{3}}\left(a_{1}^{\frac{2}{3}} a_{3}^{\frac{1}{3}}+b a_{2}^{-\frac{1}{3}} a_{1}^{\frac{1}{3}}\right) \\
& -a_{2}^{-\frac{1}{3}} a_{3}^{\frac{1}{3}}\left(a_{3}^{\frac{2}{3}} a_{2}^{\frac{1}{3}}+b a_{1}^{-\frac{1}{3}} a_{3}^{\frac{1}{3}}\right) \\
= & a_{1} a_{2} a_{3}+3 b a_{1}^{\frac{2}{3}} a_{2}^{\frac{2}{3}} a_{3}^{\frac{2}{3}}+3 b^{2} a_{1}^{\frac{1}{3}} a_{2}^{\frac{1}{3}} a_{3}^{\frac{1}{3}}+b^{3}-2 \\
& -a_{2}-b a_{1}^{-\frac{1}{3}} a_{2}^{\frac{2}{3}} a_{3}^{-\frac{1}{3}}-a_{1}-b a_{1}^{\frac{2}{3}} a_{2}^{-\frac{1}{3}} a_{3}^{-\frac{1}{3}}-a_{3}-b a_{1}^{-\frac{1}{3}} a_{2}^{-\frac{1}{3}} a_{3}^{\frac{2}{3}} \\
= & \left(a_{*}+b\right)^{3}-2-3 \bar{a}-3 b \bar{a} a_{*}^{-1} \\
= & a_{*}^{-1}\left(6 a_{*}-3 a_{*} \bar{a}-3 b \bar{a}\right)=6 a_{*}^{-1}\left(a_{*}-\bar{a}\right) .
\end{aligned}
$$

In the last line, the assumption that $a_{*}+b=2$ was applied. It follows from positivity of $\Phi_{A}$ that $a_{*} \geq \bar{a}$. Hence, $a_{*}=\bar{a}$ and consequently all $a_{i}$ are equal.

Remark 5.2 The above considerations show that (2.6) and (2.7) do not form a set of sufficient conditions for positivity of the map $\Phi_{A}$ in the case when $a_{i}$ are distinct numbers. At this stage, it is still an open problem how to generalize Theorems 1.1 and 1.2 for arbitrary numbers $a_{i}, b_{j}, c_{k}$.

Funding Information DC was supported by the Polish National Science Centre project 2015/19/B/ST1/03095, while MM was supported by Templeton Foundation Project "Quantum objectivity: between the whole and the parts". 
Open Access This article is distributed under the terms of the Creative Commons Attribution 4.0 International License (http://creativecommons.org/licenses/by/4.0/), which permits unrestricted use, distribution, and reproduction in any medium, provided you give appropriate credit to the original author(s) and the source, provide a link to the Creative Commons license, and indicate if changes were made.

\section{References}

1. Cho, S.-J., Kye, S.-H., Lee, S.G.: Generalized Choi maps in three-dimensional matrix algebra. Linear Algebra Appl. 171, 213-224 (1992)

2. Choi, M.-D.: Completely positive linear maps on complex matrices. Linear Algebra Appl. 10, 285-290 (1975)

3. Choi, M.-D.: Positive semidefinite biquadratic forms. Linear Algebra Appl. 12(2), 95-100 (1975)

4. Choi, M.D., Lam, T.Y.: Extremal positive semidefinite forms. Math. Ann. 231(1), 1-18 (1977)

5. Chruściński, D., Sarbicki, G.: Exposed positive maps in $M_{4}(C)$. Open Syst. Inf. Dyn. 19(3), 1250017 (2012). 8 pp.

6. Chruściński, D., Sarbicki, G.: Entanglement witnesses: construction, analysis and classification. J. Phys. A 47(48), 483001 (2014). 64 pp.

7. Ha, K.C.: Atomic positive linear maps in matrix algebras. Publ. Res. Inst. Math. Sci. 34(6), 591-599 (1998)

8. Ha, K.C.: Positive projections onto spin factors. Linear Algebra Appl. 348, 105-113 (2002)

9. Ha, K.C.: A class of atomic positive linear maps in matrix algebras. Linear Algebra Appl. 359, 277-290 (2003)

10. Horodecki, M., Horodecki, P., Horodecki, R.: Separability of mixed states: necessary and sufficient conditions. Phys. Lett. A 223(1-2), 1-8 (1996)

11. Jamiołkowski, A.: An effective methods of investigation of positive maps on the set of positive definite operators. Rep. Math. Phys. 5, 415-424 (1974)

12. Kye, S.-H.: A class of atomic positive maps in 3-dimensional matrix algebras. Elementary Operators and Applications. World Scientific (1992)

13. Majewski, W.A., Marciniak, M.: On a characterization of positive maps. J. Phys. A 34(29), 5863-5874 (2001)

14. Marciniak, M., Rutkowski, A.: Merging of positive maps: a construction of various classes of positive maps on matrix algebras. Linear Algebra Appl. 529, 215-257 (2017)

15. Miller, M., Olkiewicz, R.: Stable subspaces of positive maps of matrix algebras. Open Syst. Inf. Dyn. 22(2), 1550011 (2015). $16 \mathrm{pp}$.

16. Osaka, H.: Indecomposable positive maps in low dimensional matrix algebras. Linear Algebra Appl. 153, 73-83 (1991)

17. Osaka, H.: A class of extremal positive maps on $3 \times 3$ matrix algebras. Publ. Res. Inst. Math. Sci. 28(5), 747-756 (1992)

18. Osaka, H.: A series of absolutely indecomposable positive maps in matrix algebras. Linear Algebra Appl. 186, 45-53 (1993)

19. Robertson, A.G.: Schwarz inequalities and the decomposition of positive maps on $\mathrm{C}^{*}$-algebras. Math. Proc. Cambridge Philos. Soc. 94(2), 291-296 (1983)

20. Størmer, E.: Positive linear maps of operator algebras. Acta Math. 110, 233-278 (1963)

21. Størmer, E.: Decomposable positive maps on C*-algebras. Proc. Am. Math. Soc. 86(3), $402-404$ (1982)

22. Størmer, E.: Positive Linear Maps of Operator Algebras. Springer, Heidelberg (2013)

23. Tanahashi, K., Tomiyama, J.: Indecomposable positive maps in matrix algebras. Canad. Math. Bull. 31(3), 308-317 (1988)

24. Tang, W.-S.: On positive linear maps between matrix algebras. Linear Algebra Appl. 79, 33-44 (1986)

25. Woronowicz, S.L.: Positive maps of low dimensional matrix algebras. Rep. Math. Phys. 10(2), 165-183 (1976) 